MATHEMATICS OF COMPUTATION

Volume 80, Number 274, April 2011, Pages 1037-1040

S $0025-5718(2010) 02400-7$

Article electronically published on August 17, 2010

\title{
PERFECT PARALLELEPIPEDS EXIST
}

\author{
JORGE F. SAWYER AND CLIFFORD A. REITER
}

\begin{abstract}
There are parallelepipeds with edge lengths, face diagonal lengths and body diagonal lengths that are all positive integers. In particular, there is a parallelepiped with edge lengths $271,106,103$, minor face diagonal lengths 101 , 266, 255, major face diagonal lengths $183,312,323$, and body diagonal lengths $374,300,278,272$. Focused brute force searches give dozens of primitive perfect parallelepipeds. Examples include parallellepipeds with up to two rectangular faces.
\end{abstract}

\section{INTRODUCTION}

A famous open problem in Number Theory is whether there exists a perfect cuboid. That is, is there a rectangular box in $\mathbb{R}^{3}$ with positive integer edge lengths, face diagonal lengths and body diagonal lengths [2, 3]? In [2] Richard Guy poses the weaker question of whether there exist perfect parallellepipeds in $\mathbb{R}^{3}$. A perfect parallelepiped is a parallelepiped with edge lengths, face diagonal lengths and body diagonal lengths that are all positive integers. Previous attempts at finding perfect parallelepipeds focused on using rational coordinates [1, 6, 8]. Here we show that perfect parallelepipeds exist by giving examples and we describe a technique using necessary conditions within brute force searches that check at the last stage whether proposed perfect parallelepipeds can be realized in $\mathbb{R}^{3}$.

\section{THERE IS A PERFECT PARALLELEPIPED}

While we will discuss our search strategy in the next section, it is straighforward to exhibit and verify that a perfect parallelepiped exists, which is our main result. We call the shorter diagonal of a parallegram the minor diagonal and the longer diagonal the major diagonal. These will be the same for a rectangle.

Theorem 1. There is a perfect parallelepiped with edge lengths 271, 106, 103, minor face diagonal lengths 101, 266, 255, major face diagonal lengths 183, 312, 323, and body diagonal lengths 374, 300, 278, 272.

Proof. Consider the parallelepiped with edge vectors given by $\vec{u}=\langle 271,0,0\rangle, \vec{v}=$ $\left\langle\frac{9826}{271}, \frac{60 \sqrt{202398}}{271}, 0\right\rangle, \vec{w}=\left\langle\frac{6647}{271}, \frac{143754}{271} \sqrt{\frac{42}{4819}}, 66 \sqrt{\frac{8358}{4819}}\right\rangle$. Direct computation verifies that $\|\vec{u}\|=271,\|\vec{v}\|=106,\|\vec{w}\|=103,\|\vec{u}-\vec{v}\|=255,\|\vec{u}-\vec{w}\|=266,\|\vec{v}-\vec{w}\|=101$, $\|\vec{u}+\vec{v}\|=323,\|\vec{u}+\vec{w}\|=312,\|\vec{v}+\vec{w}\|=183,\|\vec{u}+\vec{v}+\vec{w}\|=374,\|\vec{u}+\vec{v}-\vec{w}\|=300$, $\|\vec{u}-\vec{v}+\vec{w}\|=278$, and $\|-\vec{u}+\vec{v}+\vec{w}\|=272$.

Received by the editor November 16, 2009 and, in revised form, December 3, 2009.

2010 Mathematics Subject Classification. Primary 11D09.

The support of a Lafayette EXCEL grant is appreciated.

(C)2010 American Mathematical Society Reverts to public domain 28 years from publication 
A Mathematica ${ }^{T M}$ script verifying those computations may be found at [7. Additional examples of perfect parallelepipeds may also be found there. These include parallelepipeds with two rectangular faces. The parallelepiped with edge vectors $\vec{u}=\langle 1120,0,0\rangle, \vec{v}=\langle 0,1035,0\rangle, \vec{w}=\left\langle 0, \frac{46548}{115}, \frac{12}{115} \sqrt{49755859}\right\rangle$ has that form. In particular, it has edge lengths $\|\vec{u}\|=1120,\|\vec{v}\|=1035$ and $\|\vec{w}\|=840$. The rectangular face diagonal lengths are $\|\vec{u}+\vec{v}\|=\|\vec{u}-\vec{v}\|=1525$ and $\|\vec{u}+\vec{w}\|=$ $\|\vec{u}-\vec{w}\|=1400$ and the other face has diagonal lengths $\|\vec{v}-\vec{w}\|=969$ and $\|\vec{v}+\vec{w}\|=1617$. The body diagonals lengths are $\|\vec{u}+\vec{v}+\vec{w}\|=\|\vec{u}-\vec{v}-\vec{w}\|=1967$ and $\|\vec{u}+\vec{v}-\vec{w}\|=\|\vec{u}-\vec{v}+\vec{w}\|=1481$.

\section{THE SEARCH}

First, we observe that the major diagonal of a parallelogram can be expressed in terms of the edges and the minor diagonal. That can be used to facilitate the search for perfect parallelograms; that is, parallelograms with edge and diagonal lengths that are all positive integers.

Lemma 2. Let $x_{1}, x_{2}$, and $d_{12}$ be positive integers with $1 \leq x_{2} \leq x_{1}$ and $x_{1}-x_{2}<$ $d_{12} \leq \sqrt{x_{1}^{2}+x_{2}^{2}}$. Then the parallelogram with edge length $x_{1}$ and $x_{2}$ and minor diagonal length $d_{12}$ is perfect if and only if $2 x_{1}^{2}+2 x_{2}^{2}-d_{12}^{2}$ is a square.

Proof. Let $\vec{u}$ and $\vec{v}$ be edge vectors for the parallelogram so that $\|\vec{u}\|=x_{1},\|\vec{v}\|=x_{2}$, $\|\vec{u}-\vec{v}\|=d_{12}$, then the result follows from observing that $\|\vec{u}+\vec{v}\|^{2}=2\|\vec{u}\|^{2}+2\|\vec{v}\|^{2}-$ $\|\vec{u}-\vec{v}\|^{2}$.

The search technique that we used determined by brute force all such $x_{1}, x_{2}$, $d_{12}$ where $x_{1}$ was below some bound. Then all non-oblique assemblies of three such perfect parallelograms with matching pairs of edges were considered as possible perfect parallelepipeds. The search was implemented in J [4].

Whether the body diagonals were of integer length was determined by the following lemma. We use $d_{i j}$ to denote the minor diagonal length of the parallelogram with edges $i$ and $j$. The body diagonal with edge $i$ having negative contribution is denoted $m_{i}, 1 \leq i \leq 3$ and $m_{4}$ denotes the length of the body diagonal when all edges contribute positively.

Lemma 3. Suppose there is a parallelepiped with edge lengths $x_{1}, x_{2}$, and $x_{3}$ and minor face diagonal lengths $d_{12}, d_{13}$ and $d_{23}$. Then the square of the body diagonal lengths are $m_{1}^{2}=-x_{1}^{2}+x_{2}^{2}+x_{3}^{2}+d_{12}^{2}+d_{13}^{2}-d_{23}^{2}, m_{2}^{2}=x_{1}^{2}-x_{2}^{2}+x_{3}^{2}+d_{12}^{2}-d_{13}^{2}+d_{23}^{2}$, $m_{3}^{2}=x_{1}^{2}+x_{2}^{2}-x_{3}^{2}-d_{12}^{2}+d_{13}^{2}+d_{23}^{2}$, and $m_{4}^{2}=3 x_{1}^{2}+3 x_{2}^{2}+3 x_{3}^{2}-d_{12}^{2}-d_{13}^{2}-d_{23}^{2}$.

Proof. Let $\vec{u}, \vec{v}, \vec{w}$ be edge vectors for the parallelepiped such that $\|\vec{u}\|=x_{1}$, $\|\vec{v}\|=x_{2},\|\vec{w}\|=x_{3},\|\vec{u}-\vec{v}\|=d_{12},\|\vec{u}-\vec{w}\|=d_{13},\|\vec{v}-\vec{w}\|=d_{23}$. Note that $2 \vec{u} \cdot \vec{v}=x_{1}^{2}+x_{2}^{2}-d_{12}^{2}$ and likewise for the other dot products. We see that

$$
\begin{aligned}
\|-\vec{u}+\vec{v}+\vec{w}\|^{2} & =\|\vec{u}\|^{2}+\|\vec{w}\|^{2}+\|\vec{w}\|^{2}-2 \vec{u} \cdot \vec{v}-2 \vec{u} \cdot \vec{w}+2 \vec{v} \cdot \vec{w} \\
& =x_{1}^{2}+x_{2}^{2}+x_{3}^{2}-\left(x_{1}^{2}+x_{2}^{2}-d_{12}^{2}\right)-\left(x_{1}^{2}+x_{3}^{2}-d_{13}^{2}\right) \\
& +\left(x_{2}^{2}+x_{3}^{2}-d_{23}^{2}\right) \\
& =-x_{1}^{2}+x_{2}^{2}+x_{3}^{2}+d_{12}^{2}+d_{13}^{2}-d_{23}^{2}
\end{aligned}
$$

as desired. The other cases are similar. 
Our search quickly located triples of perfect parallelograms with matching edge lengths $x_{1}, x_{2}$, and $x_{3}$ and minor diagonal lengths $d_{12}, d_{13}$ and $d_{23}$ that also had all four proposed body diagonals $m_{1}, m_{2}, m_{3}$ and $m_{4}$ of positive integer length. For example, the smallest such is given by $x_{1}=115, x_{2}=106, x_{3}=83, d_{12}=31$, $d_{13}=58$ and $d_{23}=75$. However, these perfect parallelograms cannot be realized as a parallelepiped in $\mathbb{R}^{3}$.

The following lemma gives the final criterion necessary for the assembly to be realizable. We let $\theta_{i j}$ denote the angle between edges $x_{i}$ and $x_{j}$ in the triangle with sides $x_{i}, x_{j}$ and $d_{i j}$ and let $c_{i j}$ denote the cosine of that angle. Note that $c_{i j}=\cos \left(\theta_{i j}\right)=\frac{x_{i}^{2}+x_{j}^{2}-d_{i j}^{2}}{2 x_{i} x_{j}}$ and by our choice of minor diagonal $0 \leq c_{i j}<1$.

Lemma 4. An edge-matched assembly of three perfect parallelograms with edge lengths $x_{1}, x_{2}$, and $x_{3}$ and minor diagonal lengths $d_{12}, d_{13}$ and $d_{23}$ can be assembled in $\mathbb{R}^{3}$ into a parallelepiped if $c_{12}^{2}+c_{13}^{2}+c_{23}^{2}<1+2 c_{12} c_{13} c_{23}$.

Proof. Let $\vec{u}=x_{1}\langle 1,0,0\rangle, \vec{v}=x_{2}\left\langle c_{12}, \sqrt{1-c_{12}^{2}}, 0\right\rangle, \rho=\frac{c_{23}-c_{12} c_{13}}{\sqrt{1-c_{12}^{2}} \sqrt{1-c_{13}^{2}}}$, and $\vec{w}=x_{3}\left\langle c_{13}, \rho \sqrt{1-c_{13}^{2}}, \sqrt{1-\rho^{2}} \sqrt{1-c_{13}^{2}}\right\rangle$. Direct computation shows that the parallelepiped generated by $\vec{u}, \vec{v}, \vec{w}$ realizes the parallelepiped with desired edges and minor diagonals provided that $-1<\rho<1$. Note that $p= \pm 1$ would yield a degenerate parallelepiped. A Mathematica ${ }^{T M}$ script verifying those computations may be found at [7. The condition that $-1<\rho<1$ is equivalent to $\rho^{2}<1$ which is equivalent to $\left(c_{23}-c_{12} c_{13}\right)^{2}<\left(1-c_{12}^{2}\right)\left(1-c_{13}^{2}\right)$ and simplifies to the required inequality.

The above lemma describes realizability using non-oblique assemblies at one vertex. Note that moving along any edge of such a perfect parallelepiped leads to a vertex with two angles becoming non-acute. Thus, configurations with an odd number of oblique angles at each vertex would be distinct from those above and these too exist [7. At least the first two of those were first found by Randall Rathbun [5].

To give some sense of the number of edge-matched non-oblique configurations checked we offer sample statisitics. When checking edges up to 3949 there were about $2 \times 10^{10}$ non-oblique edge-matched configurations tested. Of those, about $9 \times 10^{7}$ satisfied one of the necessary body diagonal conditions from Lemma 3 . About $1.7 \times 10^{6}$ satisfied two; 33403 satisfied three; 414 satisfied all four. Of those, 27 gave realizable perfect parallelipipeds.

We have established that perfect parallelepipeds exist, and some with two rectangular faces exist. The question of whether perfect cuboids exist remains open. Intermediate questions are also open. Is there a perfect parallelepiped with integer volume? Is there a perfect parallelepiped with rational coordinates?

\section{REFERENCES}

1. D. D'Argenio, C. Reiter, Families of nearly perfect parallelepipeds, JP Jour. Algebra Number Theory \& Appl. 9 (2007), 105-111. MR2407809 (2009d:11049)

2. R. Guy, Unsolved problems in number theory, Third ed., Springer, Springer-Verlag, 2004. MR2076335 (2005h:11003)

3. J. Leech, The rational cuboid revisited, Amer. Math. Monthly. 84 (1977), 518-533. Corrections 85 (1978) 472. MR0447106(56:5421)

4. Jsoftware, http://www.jsoftware.com.

5. Randall Rathbun, Private Communication. 
6. C. Reiter, J. Tirrell, Pursuing the perfecet parallelepiped, JP Jour. Algebra Number Theory \& Appl. 6 (2006), 279-274. MR2283937 (2008g:11050)

7. J. Sawyer, C. Reiter, Auxiliary materials for perfect parallelepipeds exist, http://www. lafayette.edu/ reiterc/nt/ppe/index.html.

8. J. Tirrell, C. Reiter, Matrix generations of the diophantine solutions to sums of $3 \leq n \leq 9$ squares that are square, JP Jour. Algebra, Number Theory \& Appl. 8 (2007), 69-80. MR 2370192 (2008i:11046)

Box 8681 Farinon Center, Lafayette College, Easton, Pennsylvania 18042

E-mail address: sawyerj@lafayette.edu

Department of Mathematics, Lafayette College, Easton, Pennsylvania 18042

E-mail address: reiterc@lafayette.edu 